\section{Gordonia Catheter-related Bacteremia and Native Valve Endocarditis Treated with Ceftriaxone and Oral Ciprofloxacin- A Case Report and Review of the Literature}

\section{Anupama Raghuram ${ }^{1 *}$, Stella Medina Lewis ${ }^{2}$, Rahel Bosson ${ }^{1}$ and Julio Ramirez ${ }^{1}$}

${ }^{1}$ Division of Infectious Diseases, University of Louisville, Louisville, KY, USA

${ }^{2}$ Salem Hospitalists LLC, Salem, VA, USA
We present a case of Gordonia sp Catheter-Related Bloodstream Infection (CRBSI) and native valve endocarditis in a patient with underlying cervical cancer.

\section{Case Report}

A 70-year-old female was hospitalized with intermittent fevers of four weeks duration. She had a past medical history of breast cancer (in remission) and recently diagnosed squamous cell carcinoma of the cervix, stage II-B. She had a PICC placed to receive chemotherapy with gemcitabine and cisplatin two months prior to admission. She experienced increasing episodes of intermittent fevers after PICC placement and was admitted for further management of symptoms.

Initial workup revealed positive blood culture from PICC for Pantoea agglomerans and peripheral blood culture with gram positive bacilli. The PICC line was removed on the second day of hospital admission and culture of the catheter tip also grew gram positive bacilli. The latter organism only grew at 72 hours and a gram stain determined gram positive, beaded, filamentous bacilli (Figures 1 and 2). The organism was suspected to be a Rapidly Growing Mycobacterium (RGM) based on colony morphology smooth, white to salmon colored colonies were observed (Figure 3). Fluorescent and Kinyoun stains were negative. The organism was sent for definitive identification via 16S rRNA sequencing, based on the need for accurate diagnosis. The patient was initially started on levofloxacin to cover the P. agglomerans and empiric vancomycin to cover the gram positive bacillus. Once the presumed RGM was identified during the course of the hospitalization, amikacin and azithromycin were added to the patient's antimicrobial regimen and the vancomycin was discontinued. The patient experienced pruritus and lip swelling closely associated with administration of her azithromycin dose. Azithromycin was switched to cefoxitin and levofloxacin and amikacin were continued. A CT of the abdomen and pelvis did not reveal an occult abscess. The P. agglomerans, which has been described in health care associated infections and was formerly in the Enterobacter sp group, was not felt to have a major pathogenic role, as the PICC tip and subsequent cultures never grew this organism. The patient was clinically stable and no longer having fevers at the time of discharge from the hospital.

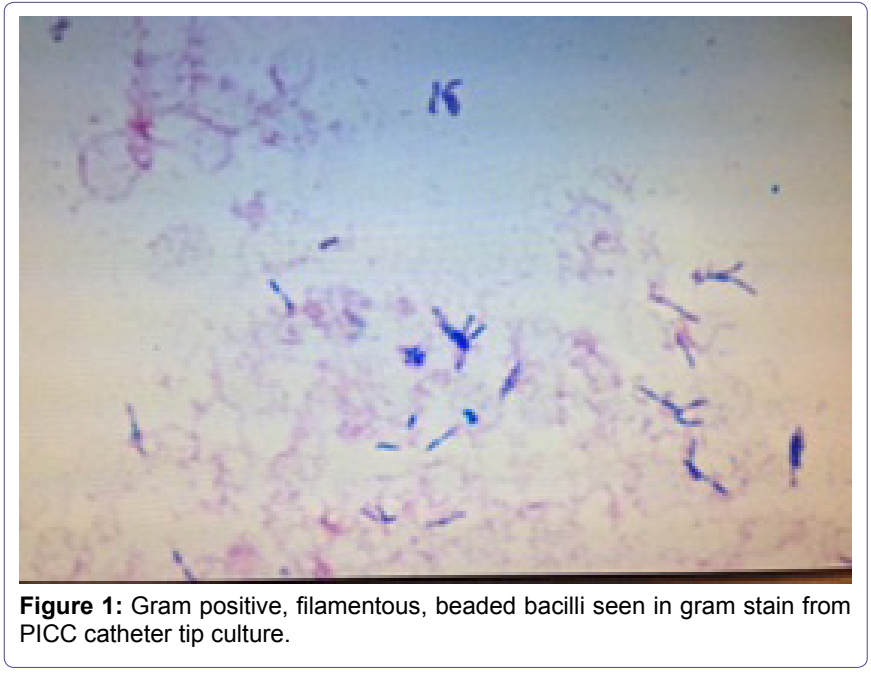




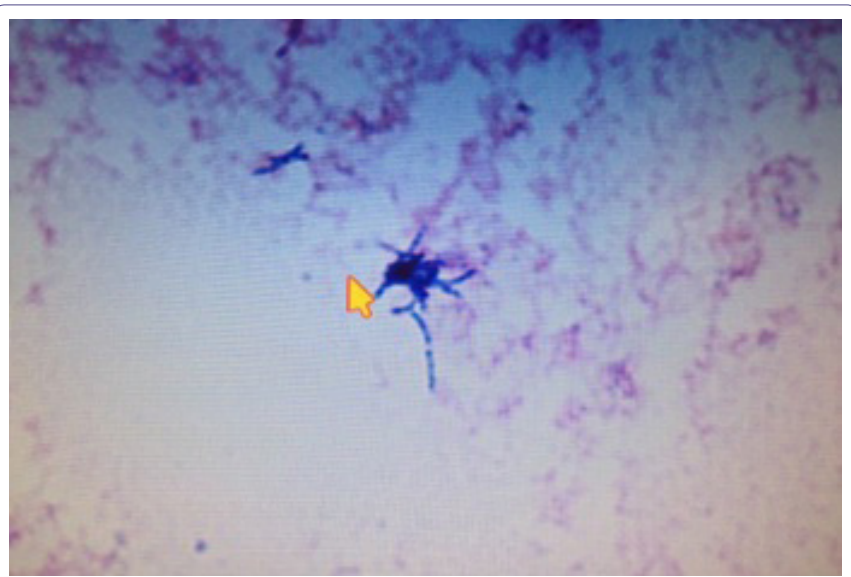

Figure 2: Cluster of thin, beaded, gram- positive bacilli seen in gram stain from PICC catheter tip culture.

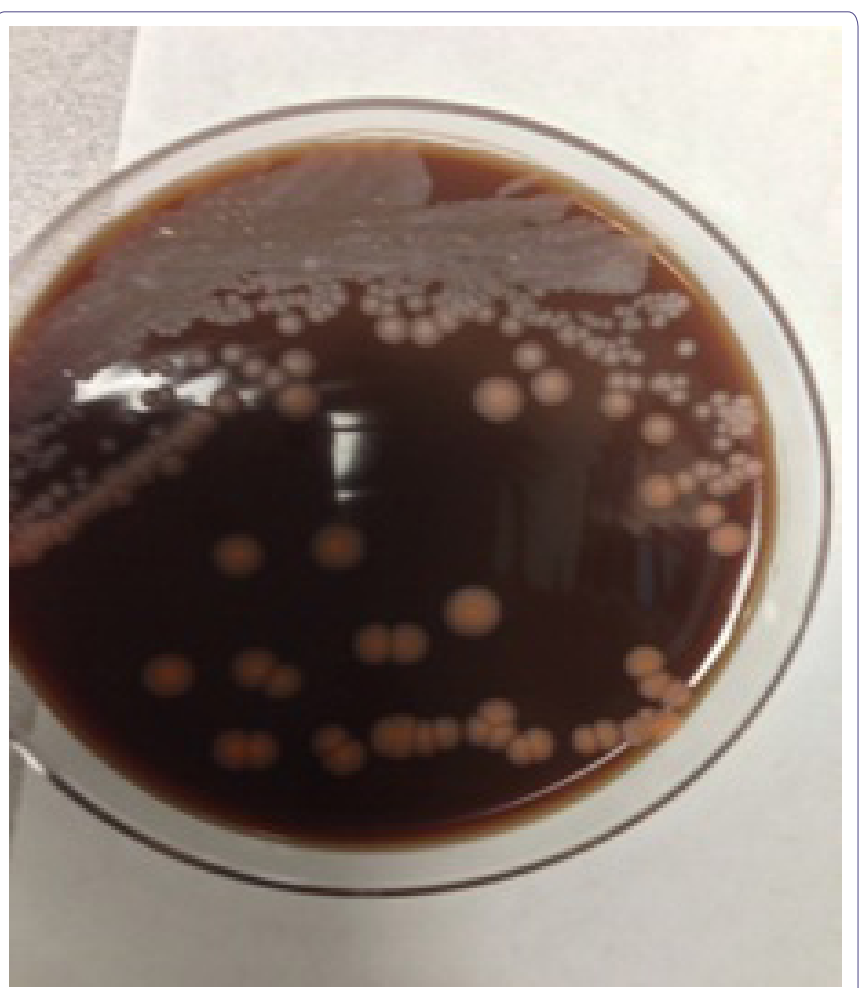

Figure 3: Colony morphology: white to salmon colored, smooth colonies observed.

Ten days after discharge, results of the 16S rRNA sequencing became available the presumed RGM was then correctly identified as Gordonia sp. The patient's infusion of amikacin had been suboptimal and, based on literature review and susceptibilities of the Gordonia isolate (susceptible to amikacin, amoxicillin-clavulanate, cefepime, ceftriaxone, ciprofloxacin, clarithromycin, imipenem, linezolid, moxifloxacin, tobramycin, trimethropim-sulfa, doxycycline, minocycline, tigecycline), her regimen was simplified to ceftriaxone and oral ciprofloxacin.

A Transesophageal Echocardiogram (TEE) was performed to assess for dissemination of infection. The TEE demonstrated a $0.45 \mathrm{x}$ $0.16 \mathrm{~cm}$ mobile, echogenic density on the left atrial side of the mitral valve, consistent with vegetation.
The patient received a total of 8 weeks of targeted antimicrobial therapy, as detailed previously. A repeat TEE close to the end of therapy showed resolution of the mitral valve vegetation. Repeat blood cultures remained negative. The patient experienced no further fevers and was back to her clinical baseline at the end of her therapeutic regimen. She passed away approximately 1 year later, secondary to progression of her cervical cancer, without evidence of recurrence of infection.

\section{Discussion}

Gordonia $s p$ are aerobic, thin, beaded gram positive bacilli [1]. They are weakly acid fast due to the presence of mycolic acid in their cell wall structure [1].

Gordonia was first proposed as a new genus by Tsukamura in 1971. Twenty-nine species of Gordonia have been described since then [3]. The organism was first isolated from water inside an automobile tire and the first clinical case was described by Kempf et al., in a bone marrow transplant recipient [4].

Genomic analysis of the organism has shown its ability to colonize indwelling catheters, due to the production of biosurfactants and biofilm. This allows for adhesion to rubber material in catheters. Gordonia sp are able to utilize rubber as their only source of carbon and represent the most potent rubber degrader tested to date [4].

Gordonia infections have been described in the immunocompromised, in patients with underlying malignancy and in those with chronic indwelling catheters. There have been cases described in immunocompetent hosts as well [5].

Grisold et al., [6] described Catheter Related Bloodstream Infection (CRBSI) and pneumonia in a 24 year old male with history of chronic anabolic steroid use. Lesens et al., [7] described the first case of Gordonia sp endocarditis in a patient with severe hemoglobinopathy, splenectomy and indwelling central venous catheter. Verma et al., [8] subsequently reported Gordonia polyisoprenivorans native valve endocarditis in a patient with underlying myelodysplastic syndrome and Osler Weber Rendu syndrome.

Four cases of Gordonia bacteremia and one case of meningitis were studied by Blaschke et al., [9] in a pediatric series. Five cases of G. terrae CRBSI, all with underlying malignancy, were identified by Pham et al., [10]. Brust et al., [11] analyzed 5 cases of Gordonia bacteremia, mostly G. terrae. Gupta et al., [12] described a 17-year-old female with underlying leukemia and Broviac catheter related Gordonia bacteremia, initially misidentified as Rhodococcus equi.

Paravertebral abscess and $G$ bronchialis bacteremia in conjunction with poorly controlled diabetes mellitus and asthma was analyzed by Sng et al., [2]. Lai et al., [13] explored 9 cases of various Gordonia infections, including CRBSI, cellulitis, traumatic wound infection and infective keratitis.

Granulomatous skin infections, brain abscesses, granulomatous mastitis and mycetoma of the hand have also been described in association with Gordonia [6].

Misidentification of Gordonia as Nocardia, Rhodococcus, Corynebacterium or non- tuberculous Mycobacteria poses a significant challenge for appropriate clinical management. A delay in diagnosis can result in dismissal as a commensal organism or in inadequate antimicrobial therapy. 
Reported susceptibility patterns are variable. In general, Gordonia $s p$ are susceptible to imipenem, ciprofloxacin, vancomycin, amoxicillin-clavulanate, amikacin and linezolid and may be resistant to tetracyclines, trimethropim- sulfamethoxazole, clarithromycin, rifampin and cefoxitin $[2-5,7,8,14]$. Duration of treatment is between 6-12 weeks [3], with catheter removal being suggested in children [8]. There are no clear guidelines for catheter removal in adults, although Lesens et al., [7] noted successful retention of central venous catheter in the setting of bacteremia and Gordonia sp native valve endocarditis after 6 weeks of intravenous antibiotic therapy.

In conclusion, there is a need for increased awareness of unusual pathogenic organisms in patients who are immunocompromised or have underlying malignancy in the setting of chronic indwelling catheters or central lines. In patients with bacteremia caused by gram positive bacilli that are difficult to identify or have atypical morphology, we suggest use of $16 \mathrm{~S}$ rRNA sequencing for definitive organism identification. Empiric initiation of a combination antimicrobial regimen, pending organism identification, such as a carbapenem or quinolone with an aminoglycoside would be most likely to address the main etiological considerations for the clinical scenario in question. Investigation for endocarditis should be considered in patients with Gordonia sp bloodstream infections. Furthermore, catheter removal should be generally encouraged in order to improve the odds of complete resolution of infection and minimize its dissemination.

\section{References}

1. Arenskötter M, Bröker D, Steinbüchel A (2004) Biology of the metabolically diverse genus Gordonia. Appl Environ Microbiol 70: 3195-3204.

2. Sng LH, Koh TH, Toney SR, Floyd M, Butler WR, et al. (2004) Bacteremia caused by Gordonia bronchialis in a patient with sequestrated lung. J Clin Microbiol 42: 2870-2871.

3. Johnson JA, Onderdonk AB, Cosimi LA, Yawetz S, Lasker BA, et al. (2011) Gordonia bronchialis bacteremia and pleural infection: case report and review of the literature. J Clin Microbiol 49: 1662-1666.
4. Moser BD, Pellegrini GJ, Lasker BA, Brown JM (2012) Pattern of antimicrobial susceptibility obtained from blood isolates of a rare but emerging human pathogen, Gordonia polyisoprenivorans. Antimicrob Agents Chemother 56: 4991-4993.

5. Langer AJ, Feja K, Lasker BA, Hinrikson HP, Morey RE, et al. (2010) Investigation of an apparent outbreak of Rhodococcus equi bacteremia. Diagn Microbiol Infect Dis 67: 95-100.

6. Grisold AJ, Roll P, Hoenigl M, Feierl G, Vicenzi-Moser R, et al. (2007) Isolation of Gordonia terrae from a patient with catheter-related bacteraemia. J Med Microbiol 56: 1687-1688.

7. Lesens O, Hansmann $\mathrm{Y}$, Riegel P, Heller R, Benaissa-Djellouli M, et al. (2000) Bacteremia and endocarditis caused by a Gordonia species in a patient with a central venous catheter. Emerg Infect Dis 6: 382-385.

8. Verma P, Brown J, Nunez VH, Morey RE, Steigerwalt AG, et al. (2006) Native valve endocarditis due to Gordonia polyisoprenivorans: case report and review of literature of bloodstream infections caused by Gordonia Species. J Clin Microbiol 44: 1905-1908.

9. Blaschke AJ, Bender J, Byington CL, Korgenski K, Daly J, et al. (2007) Gordonia species: emerging pathogens in pediatric patients that are identified by $16 S$ ribosomal RNA gene sequencing. Clin Infect Dis 45: 483-486.

10. Pham AS, Dé I, Rolston KV, Tarrand JJ, Han XY (2003) Catheter-related bacteremia caused by the nocardioform actinomycete Gordonia terrae. Clin Infect Dis 36: 524-527.

11. Brust JC, Whittier S, Scully BE, McGregor CC, Yin MT (2009) Five cases of bacteraemia due to Gordonia species. J Med Microbiol 58: 1376-1378.

12. Gupta M, Prasad D, Khara HS, Alcid D (2010) A rubber-degrading organism growing from a human body. Int J Infect Dis 14: 75-76.

13. Lai CC, Wang CY, Liu CY, Tan CK, Lin SH, et al. (2010) Infections caused by Gordonia species at a medical centre in Taiwan, 1997 to 2008. Clin Microbiol Infect 16: 1448-1453.

14. Kofteridis DP, Valachis A, Scoulica E, Christidou A, Maraki S, et al. (2012) Hickman catheter-related bacteremia caused by Gordonia sputi in a patient with breast cancer. J Infect Dev Ctries 6: 188-191. 